\section{Neuronal localization of GAS7 within human brain tissue: Implications for schizophrenia research}

\author{
Michael A. Meyer \\ Department of Neurology, Guthrie \\ Clinic, Sayre, PA, USA
}

\begin{abstract}
In view of recent data on the linkage of Gas7 protein to schizophrenia, and in view of its role in neurite outgrowth, histochemical localization of the Gas 7 protein was studied in normal human brain tissue using an online tissue atlas. Selective localization to neurons in the cerebral cortex was found with moderate levels in the hippocampus and caudate, but fairly low levels were noted within the human cerebellum and was limited to small granule cells as well as the neuropil of the cerebellar molecular layers. Despite this low intensity histochemical localization in the cerebellum, molecular data indicate a substantially large number of RNA transcripts in the cerebellum that exceeded the cerebral cortex as determined by sequencing studies.
\end{abstract}

\section{Introduction}

Recent research indicates that the growth arrest specific gene known as Gas7 gene may be a susceptibility gene for the development of schizophrenia. ${ }^{1}$ Investigators identified Gas7 as a schizophrenia risk gene in two separate Chinese populations; they also found that Gas7 overexpression increased branching of leading processes within nerve cells, thereby arresting neuronal migration, while suppression of Gas7 could inhibit the process; furthermore,Gas7-deficient mice showed behavioral changes with sensorimotor gating deficits. ${ }^{1}$ Early basic science studies dating back to 1998 demonstrated robust histochemical staining within the mouse brain mainly within the cortex, hippocampus and cerebellum with less intense expression in the caudate; overexpression in neuroblastoma cells induced neurite extension whereas inhibition of Gas 7 production inhibited neurite outgrowth from mouse cerebellar Purkinje cells. ${ }^{2}$

Therefore, in light of new data on the link to both neuronal development and schizophrenia, expression within normal human tissues was therefore conducted.
Immunohistochemical localization of Gas7 was examined in normal human brain tissue to compare regional expression patterns and to study gene expression at the cellular level.

\section{Materials and Methods}

The Human Protein Atlas was used for this study (antibody: HPA004838). Histochemical expression in normal brain tissue was studied using the open access online atlas; regional quantitative gene transcription data in RPKM units was also analyzed (website address: https://www.proteinatlas.org/). Three-dimensional structure of the Gas 7 protein was also studied using the National Center for Biotechnology online tool for structural displays of protein molecules (https://www.ncbi.nlm.nih.gov/ Structure/pdb/2LX7.

\section{Results}

The three-dimensional structure of a 60 amino acid fragment from the full-length molecule of 416 amino acids is shown in figure one. As shown in figure two, Gas7 was localized to the cell bodies of medium sized neurons in the hippocampus, cerebral cortex, and basal ganglia as well as small granule cells of the cerebellum; lighter staining was found within glial cells. Neuronal positivity seemed somewhat more intense for cortical neurons but the distribution was fairly equitable amongst cortical samples as compared to hippocampus and caudate. Human cerebellar Purkinje neurons were conspicuously negative but light staining was seen in the cerebellar granule cells and molecular layer neuropil. The number of RNA transcripts was significantly higher in the cerebellum as determined by RPKM measurements from RNA sequencing of the tissue ( 85.2 for the cerebellum versus 44.4 for the cortex). Of additional note, the expression of Gas7 appeared to be limited to the cytoplasm in the caudate whereas cortical neurons had diffuse localization in the nucleus and cytoplasm. The cerebral cortex neurons displayed the most intense localization pattern within neurons.

\section{Discussion}

As shown here, Gas7 expression within the brain is mainly within neurons of the cerebral cortex but can be seen within the caudate and hippocampus as well. The protein has been known to be preferentially
Correspondence: Department of Neurology, Guthrie Clinic, 130 Centerway, Corning, NY 14830, USA.

Tel.: +1.607.973.8000

E-mail: michaelandrewmeyer@gmail.com

Key words: Gas7; Schizophrenia; isoforms; gene; neurite.

Acknowledgements: the author acknowledges the help of the Human Protein Atlas project from Sweden (website address: https://www.proteinatlas.org/) from a publically accessible online tissue atlas and no need for institutional review board review. The author wishes to thank the following researchers for reviewing this manuscript: Michal K. Stachowiak and Dost Ongur.

Conflict of interest: the author declares no potential conflict of interest.

Funding: none.

Received for publication: 3 August 2018.

Accepted for publication: 16 November 2018.

This work is licensed under a Creative Commons Attribution NonCommercial 4.0 License (CC BY-NC 4.0).

(C) Copyright M.A. Meyer, 2018

Licensee PAGEPress, Italy

Neurology International 2018; 10:7563

doi:10.4081/ni.2018.7563

expressed within brain tissue in the brain and has key roles in neurite outgrowth. As Gas7 binds to the terminal part of actin microfilament, it is a necessary factor for the process of actin induced outgrowth to take place and form cellular extensions of lamellopodia and filopodia. ${ }^{3}$

Two isoforms of the protein have been found with differential expression in the human brain, with one type being 2,427 nucleotides in length and known as hGas7a; this isoform is homologous to an isoform in the mouse that is mainly found in the cerebellum. Isoform hGas7-a induces formation of small lamellipodia extensions when expressed ectopically in neuroblastoma cells, whereas the longer hGas7-b isoform measuring 2,610 nucleotides is 14 times less frequent and induces small filopodia in ectopic expression models. ${ }^{3}$ The encoded protein has been found by tissue culture studies to promote neurite outgrowth. ${ }^{3}$ The clinical implications of these basic science studies might be that inadequate levels of Gas7 might therefore alter normal patterns of neurite outgrowth and patterns of neuronal connectivity in human brain development; with mutations and deletions in the Gas7 gene, diminished neural outgrowth in development may possibly 
contribute to long term alterations in neuronal connectivity and possibly contribute to schizophrenia symptomatology.

Of additional note, studies from 2010 showed that specific interaction between

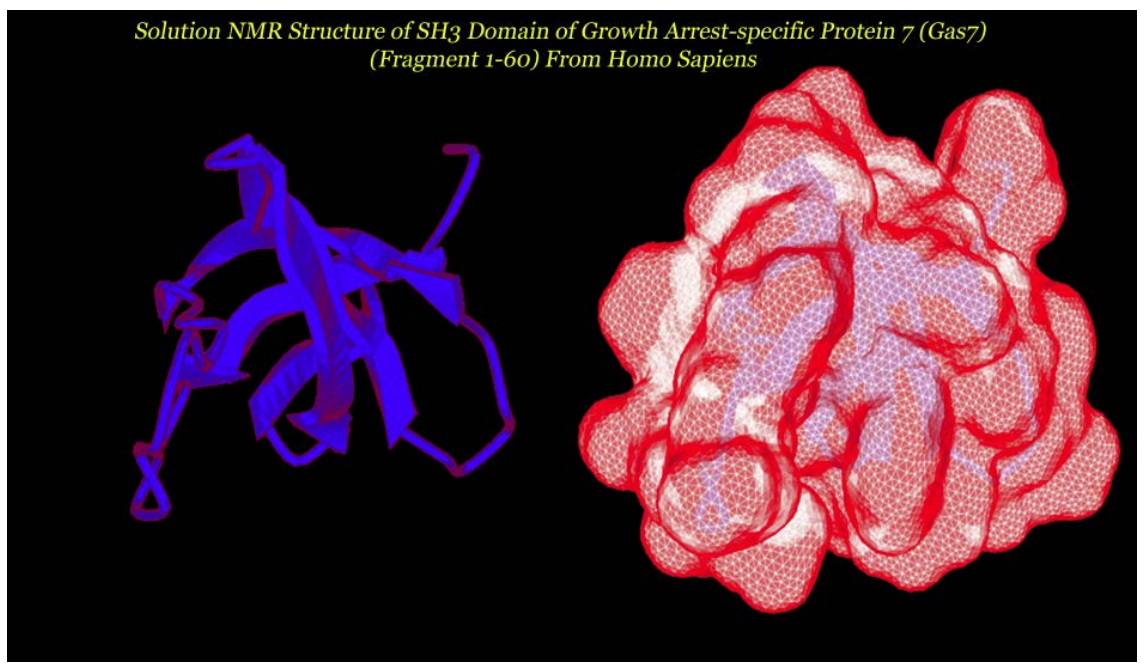

Figure 1. Three-dimensional structure of the 1-60 fragment from the $\mathbf{4 1 6}$ amino acid protein showing the secondary beta sheet structure at left versus the tertiary surface three-dimensional structure at right. The full 416 amino acid molecule appears to be involved with neuronal development and can stimulate neurite outgrowth when overexpressed within neuroblastoma cells.

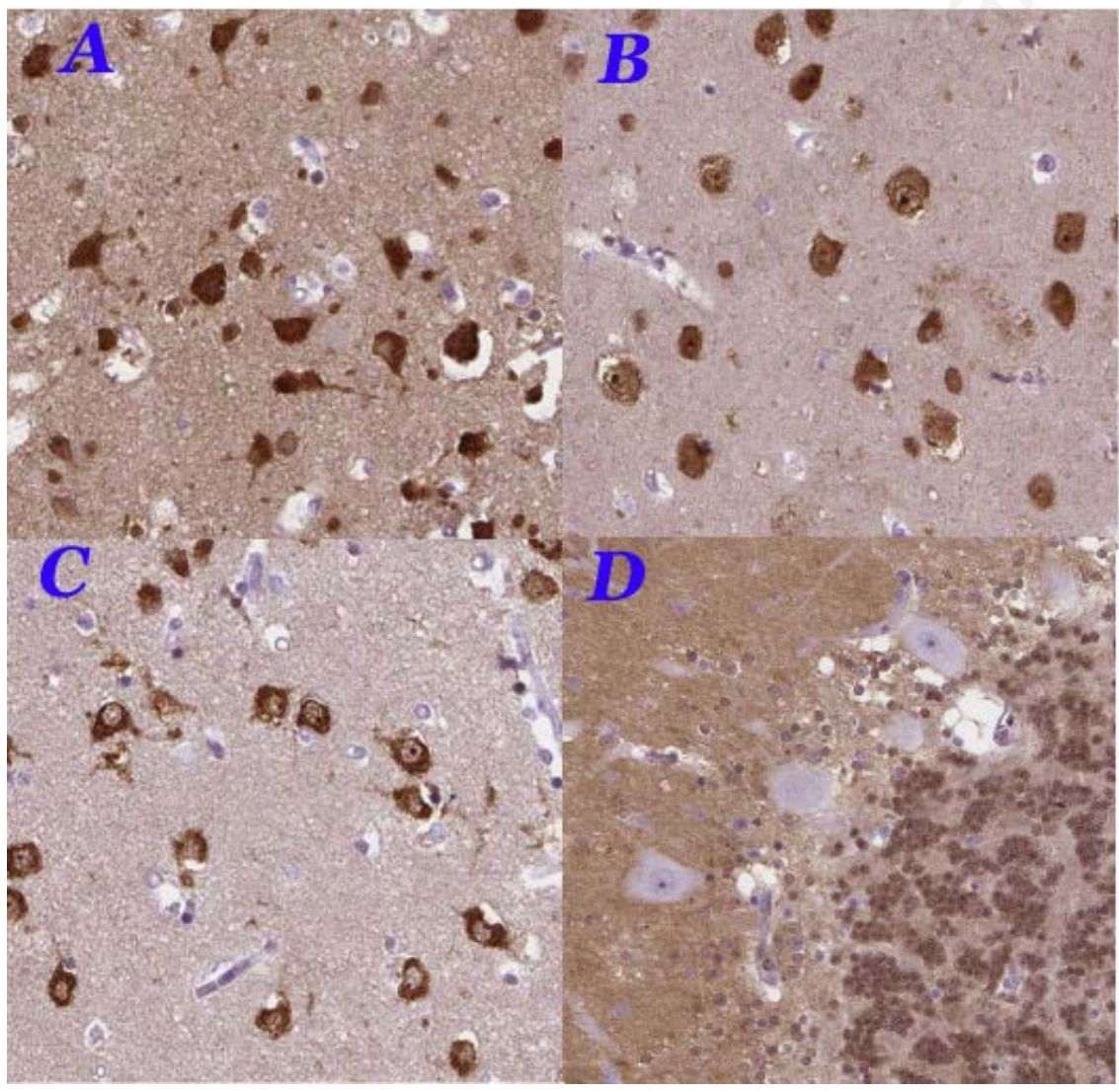

Figure 2. A) normal cerebral cortex, female age 52: mean RPKM 44.4; $\max 75.5$. B) normal hippocampus male age 58: mean RPKM 30.9; $\max$ 63.0. C) normal caudate male age 54: mean RPKM 38.5; $\max 80.9$. D) normal cerebellum, female age 54: mean RPKM 87.2, $\max 161.0$. tion/differentiation. This protein is also known as Neural-Wiskott Aldrich Syndrome Protein and is a critical regulator of actin dynamics that regulates Arp $2 / 3$ which is the actin-related protein 2 and 3.4

In view of well described developmental anomalies of the brain including disrupted cellular organization within the temporal lobes of schizophrenic patients including reports of heterotopic or abnormally positioned pre-alpha neuron clusters within the entorhinal cortex, 5 it is hypothesized that mutations or micro-deletions within the Gas7 gene may lead to abnormal neuronal development with altered connectivity to generate a pathologic framework that predisposes affected individuals to schizophrenic symptoms. Further neuropathologic correlative work is needed along with further study of animal models that may include Gas7 knockouts.

\section{Conclusions}

It remains unknown if the regional distribution of Gas7 varies in post-mortem brain tissue from patients with schizophrenia. Further research is needed on this as well as to determine if the cellular localization pattern described here varies in schizophrenia; more research is clearly needed.

\section{References}

1. Zhang Z, Zheng F, You Y, et al. Growth arrest specific gene 7 is associated with schizophrenia and regulates neuronal migration and morphogenesis. Mol Brain 2016;9:54.

2. Ju YT, Chang AC, She BR, et al. gas7: A gene expressed preferentially in growth-arrested fibroblasts and terminally differentiated Purkinje neurons affects neurite formation. Proc Natl Acad Sci USA 1998;95:11423-8.

3. Chao CC, Chang PY, Lu HH. Human Gas7 isoforms homologous to mouse transcripts differentially induce neurite outgrowth. J Neurosci Res 2005;81: 153-62.

4. You JJ, Lin-Chao S. Gas7 functions with N-WASP to regulate the neurite outgrowth of hippocampal neurons. J Biol Chem 2010;285:11652-66.

5. Honer WG, Bassett AS, Falkai P, et al. A case study of temporal lobe development in familial schizophrenia. Psychol Med 1996;26:191-5. 